\title{
The effect of rotation on configural encoding in a face-matching task
}

\author{
Andrew J Edmonds $\uparrow$, Michael B Lewis \\ School of Psychology, Cardiff University, Tower Building, Park Place, Cardiff CF10 3AT, Wales, UK; \\ e-mail: EdmondsA@cardiff.ac.uk \\ Received 7 October 2005, in revised form 31 May 2006; published online 7 March 2007
}

\begin{abstract}
Inversion disrupts encoding of faces because of the disruption of configural encoding as evident in the Thatcher illusion (Thompson 1980, Perception 9 483-484). Here we consider the effect of rotation on the loss of configural encoding in a same/different matching paradigm. Participants decided whether two faces were of the same type (both normal or both Thatcherised) or not, at five angles of rotation $\left(0^{\circ}, 45^{\circ}, 90^{\circ}, 135^{\circ}, 180^{\circ}\right)$. When the faces were both of the same person, the disruption due to rotation for 'same-type' judgments was linear and approximately equal for normal and Thatcherised face pairs. In experiment 2, with different-person face pairs, the effect of rotation was much greater for Thatcherised face pairs than for normal face pairs. These findings are in contrast to those reported by Boutsen and Humphreys (2003, Quarterly Journal of Experimental Psychology A 56 955-975), and reasons for these differences, along with implications, are discussed.
\end{abstract}

\section{Introduction}

A considerable amount of research has shown that inverting a face disrupts the recognition of that face-an effect which is disproportionate to that of inverting other objects, such as houses or aeroplanes (Yin 1969). There is a variety of evidence to suggest that it is information about the configuration of facial features (their relative arrangement to each other within a face) that is disrupted by inversion, and that inversion is more disruptive to the processing of configural information than to that of featural information. Searcy and Bartlett (1996) found effects of inversion on a simultaneouscomparison task with spatially distorted and featurally distorted faces. Inversion significantly hindered participants' ability to decide, within a given time frame, that a pair of spatially distorted faces were the same or different; but this effect was not found with featurally distorted pairs, and responses made within this time feature $(3 \mathrm{~s})$ were longer for detecting configural differences than for detecting featural changes. There is, therefore, evidence to suggest that the processing of upright, normal faces is largely dependent on configural processing, whereas inverted faces are thought to require a more featural means of processing (see also Bartlett and Searcy 1993; Rhodes et al 1993; Lewis and Johnston 1997).

It is important to note that, although there is a wide range of evidence to support the notion that two types of encoding-configural and featural-are involved in face perception, a number of different terms have been used to refer to different definitions of these types of information. Terms such as 'second-order relational information', 'configural' information, and 'holistic' information have referred to configural information as being the combination of components that make up an individual face (eg Sergent 1984), or the configuration formed by the individual arrangements of facial features (eg Diamond and Carey 1986; Bartlett and Searcy 1993). Nevertheless, featural information is generally regarded as the presence of a particular feature or type of feature and the encoding of these parts independent of their context (Diamond and Carey 1986), whereas configural information is gained from the relative arrangement of

$\uparrow$ Current address: Department of Psychology, University of Kent at Canterbury, Canterbury, Kent CT2 7NP, UK; e-mail: A.J.Edmonds@kent.ac.uk 
features to each other within a face. Lewis and Glenister (2003) note, however, that this is not a clear-cut distinction, as the presence of a certain feature, such as a wide mouth, might be regarded as featural information, but only if it is wide in relation to a particularly thin face can it be regarded as a configural feature. Indeed, configural information might best be operationally defined as that which is disrupted by inversion (Lewis 2001).

Many researchers have considered the detrimental effect of inversion on face perception, but until recently few had looked at the effect of other (intermediate) angles of rotation. An influential account of why configural information is difficult to retrieve from inverted faces is that rotated faces need to be mentally reoriented to the upright before they can be recognised, and inverted faces are particularly hard to recognise because we are unable to simultaneously reorient the features of faces (Rock 1973). The need to rotate each of the features one at a time means that configural information about their spatial relationships cannot be accessed (see also Carey and Diamond 1977). Indeed, in an object-recognition study, Shepard and Metzler (1971) found that, when participants had to decide whether two simultaneously presented shapes were the same or different, reaction times (RTs) showed a linear increase as a function of the difference in orientation between the two shapes.

Face-rotation experiments have been used to assess the predictions of this mental-rotation hypothesis. If the face-inversion effect is due to the limitations of a face-normalisation mechanism, there are three possible predictions. The first is that the failure of the normalisation process for configural information is total, so faces can only be processed configurally if shown at views for which face representations are stored. Second, it may be possible to mentally rotate whole faces which are only slightly rotated from the upright, but faces rotated beyond a certain point may require the rotation of individual features, bringing about a change in processing strategy between upright and inverted faces (Valentine and Bruce 1988). Finally, recognition performance may depend on the amount of normalisation required. The first two scenarios would predict a nonlinear effect of rotation, whereas the third hypothesis suggests a linear relationship.

Schwaninger and Mast (2005) have recently provided a direct test of Rock's (1973) mental-rotation hypothesis. Using a similar same/different matching task to Valentine and Bruce's (1988), they asked participants to detect featural or configural changes in seven angles of orientation from upright to inverted $\left(0^{\circ}, 30^{\circ}, 60^{\circ}, 90^{\circ}, 120^{\circ}, 150^{\circ}, 180^{\circ}\right)$. The mental-rotation hypothesis argues that rotated faces can only be processed by the mental rotation of features, so rotation should affect the accuracy of configural changes but not of featural changes. This mental-rotation process is time consuming, however, and therefore RTs will be affected by rotation both for featural and for configural changes. For same trials (ie when there was no change to be detected), the hypothesis predicts no difference between the two conditions, because trials always contain identical stimuli. Participants would therefore mentally rotate the parts to verify that the two faces are the same, and this would be the case in both conditions. The findings for both types of changes were consistent with this prediction. For different trials, the accuracy of the detection of featural changes was not affected by rotation of the face, but configural changes were dramatically affected by face rotation and, moreover, the RT data showed that featural and configural changes were equally affected by face rotation. For same trials, there was no difference between the component and configural conditions in terms of accuracy or reaction times.

Schwaninger and Mast (2005) did not, however, find a linear relationship between detection of changes (different trials) and angle of rotation. The error data revealed that participants made the greatest number of errors for faces rotated $90^{\circ}$ and $120^{\circ}$ from upright, with this number dropping for faces rotated $150^{\circ}$ and inverted faces. 
Indeed, a similar, albeit much less pronounced, pattern was found for the RT data, with $90^{\circ}$ and $120^{\circ}$ rotations yielding longer RTs than $150^{\circ}$-rotated or inverted faces. Schwaninger and Mast (2005) did not test the significance of these findings, or the linearity of these sets of data, but did suggest that a mental 'flipping' strategy, proposed by Corballis et al (1978), may be being used for inverted faces, whereby participants flip inverted faces to match them to a stored representation of that face. Such an explanation would account for the nonlinear effects of rotation as shown by greater accuracy (and slightly faster RTs) in detecting configural changes in inverted faces than in faces shown at intermediate angles of orientation.

The role of configural information in the processing of upright and inverted faces has also been considered with the use of the Thatcher illusion (Thompson 1980). The Thatcher illusion is an illusion where the eyes and mouth are inverted within an upright face, resulting in a grotesque image. The illusion is that, if the whole image is then inverted, the changes made to the face are disrupted and the face no longer appears abnormal or gruesome. This illusion is thought to result from the disruption of configural information produced by inverting the face parts relative to their surrounding context. That is, upright faces are more sensitive to (changes in) configural processing (eg Bartlett and Searcy 1993; Rhodes et al 1993), whereas with inverted faces the process of inversion has already disturbed this configural information, making configural processing less efficient.

Boutsen and Humphreys (2003) considered the processing of Thatcherised faces within a same/different matching paradigm. Participants were asked to decide whether the features of two faces were the same (both normal or both Thatcherised) or not (one normal, one Thatcherised), in both upright and inverted orientations. Both faces were the same image of the same person on any one trial. Boutsen and Humphreys used RTs to decide that face pairs were the same (both normal or both Thatcherised), with both same-identity and different-identity face pairs. Same-identity pairs, where the two images are identical, can be based on a comparison of individual features, and thus, while still available, configural information becomes less useful in this task, making the matching of individual features more likely. With different-identity pairs, however, matching cannot be based on individual features, as the two images are always of different people, and so matching will therefore require information about the orientation of face parts relative to their context in order to complete the task. For same pairs, an inversion effect was found for normal but not for Thatcherised faces. This was the case both for same-identity (experiment 3) and for different-identity pairs (experiment 4). For different pairs, responses to inverted faces were significantly slower than those to upright faces (see also Lewis and Johnston 1997). For same-identity parts (experiment 1), Thatcherised pairs again yielded longer RTs than normal pairs, but there was no effect of inversion for normal or Thatcherised parts. For differentperson face parts (experiment 2), Thatcherised pairs again yielded longer RTs than normal pairs.

Boutsen and Humphreys (2003) suggest that the presence of the face context in experiment 3 influenced encoding of the face parts, enabling the coding of these features as part of a configuration. For normal faces, the whole-face context facilitated a configural encoding strategy, even when a feature-based strategy is encouraged. This can explain why an inversion effect was obtained with whole, normal faces but not with Thatcherised faces. In contrast, in experiments 2 and 4 we found an inversion effect for normal parts and normal whole faces, suggesting that the additional face context does not influence or facilitate configural encoding when matching cannot be image-based. It therefore appears that local configural processing, which is independent of the face context, was used in this task and that this information is disrupted by inversion and Thatcherisation. 
The absence of inversion effects for Thatcherised face pairs, on the other hand, suggests that the presence of the face context in experiments 3 and 4 may have disturbed configural processing, particularly with upright faces, as this renders the context and the Thatcherised parts incompatible (Boutsen and Humphreys 2003). Thus it would appear that configural processing occurs at a more global level for Thatcherised faces; processing of Thatcherised faces involves the encoding of spatial relations and their relationship to the whole face context. Boutsen and Humphreys (2003) argue that, taken as a whole, these findings suggest that the encoding of Thatcherised faces is qualitatively different from that of normal faces.

In recent years a number of authors have looked at the effects of rotation on perception of the Thatcher illusion. Murray et al (2000) found that there was a discontinuity in bizarreness ratings of Thatcherised faces between $90^{\circ}$ and $120^{\circ}$ rotations, suggesting that there may be a decrease in the utility of configural information as the face is rotated from the upright view. Lewis (2001) considered RTs to simply decide that rotated faces were (or were not) Thatcherised, and found a nonlinear increase in RTs as the face was rotated away from upright.

Here we attempt to combine the methodologies of Boutsen and Humphreys (2003) and Schwaninger and Mast (2005) to look more closely at the effect of rotation on the loss of configural information on a simultaneous-matching task. We look at the effect of rotation on performance in a same/different matching task with Thatcherised and normal faces both with same-identity pairs (which encourages a featural-processing strategy) in experiment 1 and with different-identity pairs (which will encourage a more configural-processing strategy) in experiment 2 to examine why configural information is difficult to retrieve from inverted faces. Particular reference will be made to the different predictions of the mental-rotation hypothesis, and also concerning the linear or nonlinear nature of the relationship between rotation and face recognition.

\section{Experiment 1}

Previous research with a simultaneous-matching task suggests that we should expect to find a greater detrimental effect of rotation for normal faces than for Thatcherised faces on this type of task, regardless whether the images are same-person pairs or different-person pairs (Boutsen and Humphreys 2003). However, if, as has been suggested, same-person matching does encourage a featural-processing strategy, we might expect to see similar effects of rotation for normal and Thatcherised faces. Such a pattern of results would also be predicted by the mental-rotation hypothesis. Indeed, although there are a number of methodological differences between our study and that of Schwaninger and Mast (2005), if Thatcherised faces can be compared to their configurally distorted faces, we may expect to find similar effects of rotation. In their study, when the two faces were the same, there was a significant effect of rotation, but this was roughly the same for configurally and featurally altered face pairs. Thus, we may expect to observe a similar pattern of results on same trials, with a significant, nonlinear effect of rotation for same normal and Thatcherised pairs, but no difference between the two.

We also look closely at the effect of rotation on different trials in this simultaneousmatching task, which has not previously been considered. We would expect a clear effect of inversion in this task because, as noted earlier, an inverted Thatcherised face and an inverted normal face look very similar to each other. Considering the intermediate angles of rotation, however, may also inform us whether the two faces become progressively more similar over rotation - as may be indicated by a linear increase in RTs - or whether there is a definite point at which processing of the two faces becomes similar - in which case a sharp increase in RT would be expected. Schwaninger and Mast (2005) found evidence consistent with an abrupt change in processing in the detection 
of configural changes in a sequential matching task. Participants made the greatest number of errors for faces rotated $90^{\circ}$ and $120^{\circ}$ from upright, with this number dropping for $150^{\circ}$ rotated and for inverted faces, with a similar pattern found for RTs. Thus, on different trials we may expect to find a definite switch in accuracy rates and RTs as the faces are rotated away from the upright.

\subsection{Method}

2.1.1 Design. The two within-participants independent variables were the orientation of the faces [upright $\left(0^{\circ}\right), 45^{\circ}, 90^{\circ}, 135^{\circ}$, and $180^{\circ}$ ] and whether the two faces were the same or different (both normal, both Thatcherised, or one normal and one Thatcherised). The dependent variables were the accuracy and latency of responses to the same/different decision for same face pairs.

2.1.2 Participants. Twenty undergraduates from Cardiff University, with normal or corrected-to-normal vision, received course credits for their participation in this experiment.

2.1.3 Materials. Six full-frontal, colour faces (four male, two female) obtained from the Psychological Image Collection at Stirling (PICS; http://pics.psych.stir.ac.uk) were used in this experiment. Each face was of approximately similar size, and each image was resized to measure $283 \times 346$ pixels on the screen. The images were centered vertically on the screen, and were 500 pixels apart horizontally. The eyes and mouth were selected and inverted separately for each face. The normal and Thatcherised upright faces were then rotated through the remaining four angles $\left(45^{\circ} .90^{\circ}, 135^{\circ}\right.$, and $\left.180^{\circ}\right)$. The faces were used to create a set of 120 face pairs, 60 for each type of response (same/different), 12 for each angle of rotation. Each face pair was repeated three times, making 360 trials in the experiment. On 'different' trials (when a normal and a Thatcherised face were paired together), the position of the normal face was counterbalanced between trials, such that it appeared on the left of the screen (and the Thatcherised face on the right) in half of the trials, and on the right of the screen (with the Thatcherised face on the left) in the other half. All images were presented on a computer with a screen resolution of $800 \times 600$ pixels with Superlab, and subtended a visual angle of approximately $5 \mathrm{deg}$. Examples of the stimuli used in the two experiments are shown in figure 1.
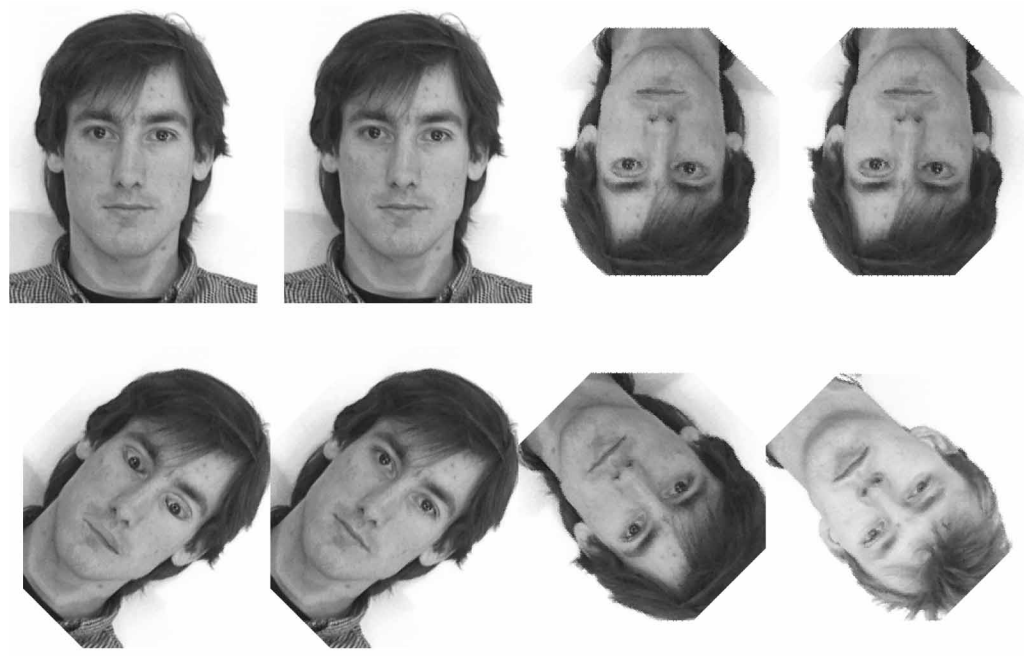

Figure 1. Examples of the stimuli used in the two experiments. The top row shows same-person normal and Thatcherised face pairs (upright and inverted). The bottom row shows a 'different' pair (one normal, one Thatcherised) and a different-person pair (both of the same type). 
2.1.4 Procedure. In each trial, participants saw two faces on the screen. These faces were either both normal, both Thatcherised, or one normal and one Thatcherised. Participants were asked to indicate whether they thought the two images were the same or different, by pressing one of two keys on a keyboard. In each trial, the face pair was presented until the participant made a response, followed by a $1 \mathrm{~s}$ blank interval before the next trial. Participants were asked to respond as quickly and as accurately as possible.

\subsection{Results}

Separate analyses were performed for the same and different pair trials. Only RTs for correct responses and for those not exceeding three standard deviations from the mean RT for each participant were analysed. The mean correct RTs for responses to normal and Thatcherised faces, and the percentage of errors for each type of face, were calculated for each angle of rotation. On same-pair trials, an error was a 'different' response to a pair of normal faces or a pair of Thatcherised faces, while on different-pair trials, an error was a 'same' response to a pair consisting of a normal face and a Thatcherised face. The mean RTs for same pairs are shown in figure 2.
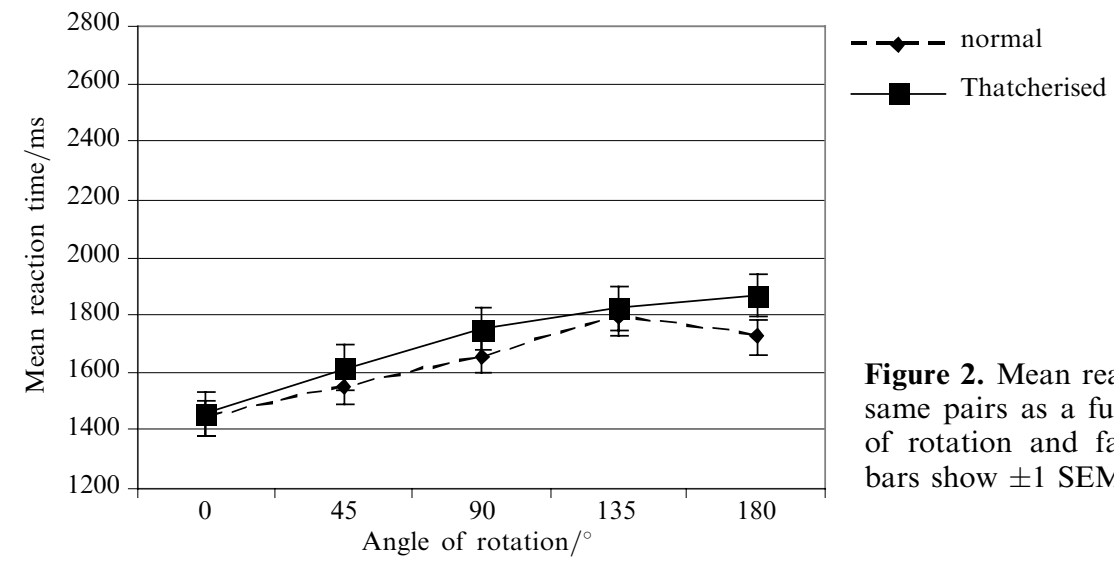

Figure 2. Mean reaction times for same pairs as a function of angle of rotation and face type. Error bars show \pm 1 SEM.

A repeated-measures ANOVA was conducted on the mean RTs shown in figure 2. There was a significant effect of orientation on performance $\left(F_{4,76}=11.667, p<0.05\right)$, but no significant effect of face type $\left(F_{1,19}=2.452, p>0.05\right)$ and no significant interaction between orientation and face type $\left(F_{4,76}=0.699, p>0.05\right)$. There was a slight trend for a decrease in RTs for normal faces rotated between $135^{\circ}$ and $180^{\circ}$, while Thatcherised faces showed a continuous increase over the same distance, but the decrease for normal faces was not significant $\left(t_{19}=1.626, p>0.05\right)$, and the decrease for normal faces did not produce a significant difference between normal and Thatcherised faces in the inverted condition $\left(t_{19}=1.299, p>0.05\right)$. These results suggest that rotation had a detrimental effect on same-pair face-matching performance, and this effect was approximately the same for normal and Thatcherised faces.

A repeated-measures ANOVA was conducted on the error data shown in figure 3. There was a significant effect of both rotation $\left(F_{4,76}=2.955, p<0.05\right)$ and face type $\left(F_{1,19}=23.699, p<0.05\right)$, with a detrimental effect of rotation away from upright, and Thatcherised face pairs producing more errors than normal face pairs. There was no significant interaction between rotation and face type $\left(F_{4,76}=1.420, p>0.05\right)$. Separate one-factor within-subjects ANOVAs showed a main effect of rotation for normal pairs $\left(F_{4,76}=3.451, p<0.05\right)$, but not for Thatcherised pairs $\left(F_{4,76}=1.753\right.$, $p>0.05)$. The difference between normal and Thatcherised face pairs was only significant in the upright $\left(t_{19}=3.387, p<0.05\right)$ and inverted $\left(t_{19}=3.907, p<0.05\right)$ orientations. 


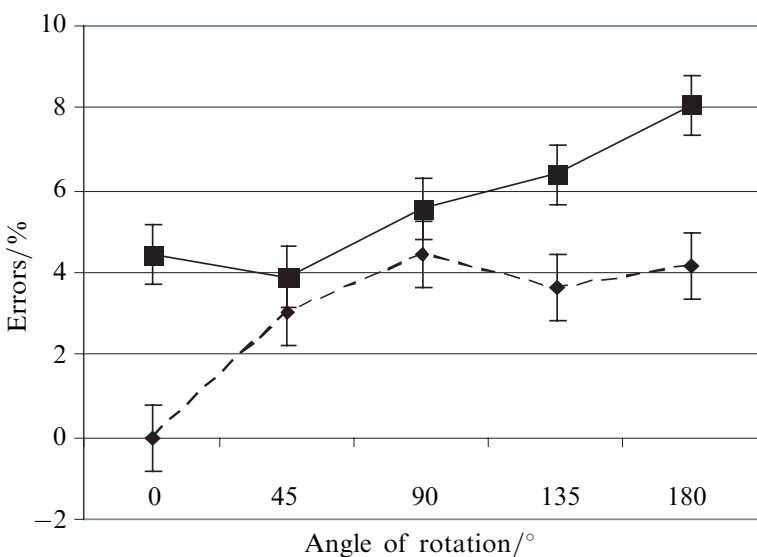

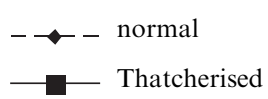

Figure 3. Percentage of errors for same pairs as a function of angle of rotation and face type. Error bars show \pm 1 SEM.

The mean RTs and percentage of errors for different pairs are shown in figures 4 and 5, respectively. A repeated-measures ANOVA showed a significant effect of rotation on RT performance $\left(F_{4,76}=17.673, p<0.05\right)$. A series of paired-sample $t$-tests revealed significant differences between $0^{\circ}$ and all other angles of rotated faces, and between each angle of rotation (ie between $0^{\circ}$ and $45^{\circ}, 45^{\circ}$ and $90^{\circ}, 90^{\circ}$ and $135^{\circ}$, and $135^{\circ}$ and $180^{\circ} ; p<0.05$ in all cases). Figure 4 shows a steady increase in RTs for rotations between $0^{\circ}$ and $90^{\circ}$, with a trend for a sharp increase in RTs between $90^{\circ}$ and $135^{\circ}$; but a paired-samples $t$-test revealed that this increase in RTs was not significantly larger than that for rotations between $0^{\circ}$ and $90^{\circ}\left(t_{19}=1.031, p>0.05\right)$, or larger than that for rotations between $45^{\circ}$ and $90^{\circ}\left(t_{19}=1.765, p>0.05\right)$, suggesting that for RTs there is a progressive effect of rotation on this task.
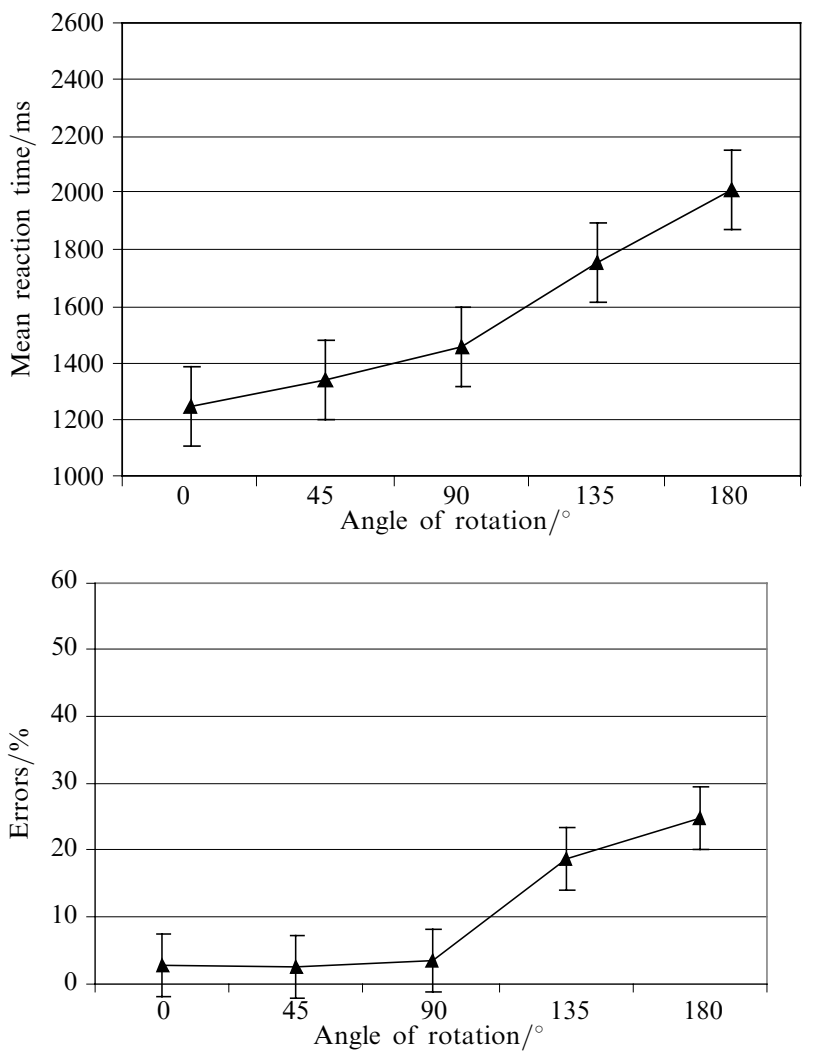

Figure 4. Mean reaction time for different pairs as a function of angle of rotation. Error bars show \pm 1 SEM.
Figure 5. Percentage of errors for different pairs as a function of angle of rotation. Error bars show \pm 1 SEM. 
A repeated-measures ANOVA of the error data (figure 5) also revealed a significant effect of rotation $\left(F_{4,76}=28.839, p<0.05\right)$, but showed an abrupt increase in errors for faces rotated beyond $90^{\circ}\left(t_{19}=5.792, p<0.05\right)$. The increase in errors between $135^{\circ}$ and $180^{\circ}$ was also significant $\left(t_{19}=2.666, p<0.05\right)$. These analyses suggest that RTs to detect that one face in an otherwise identical pair is Thatcherised becomes progressively longer with increasing rotation away from the upright, with a significant increase in RTs between each angle of rotation. However, in terms of accuracy there is a clear point of rotation beyond which participants find it difficult to detect that the two faces are different.

\subsection{Discussion}

The results of this experiment show a number of differences with previous research. Boutsen and Humphreys (2003) found that the correct matching of two Thatcherised faces yielded significantly longer RTs than did the matching of two normal faces. Although this was also true of our findings, the difference between normal and Thatcherised faces was not significant in our study. Moreover, a significant effect of rotation was found for both normal and Thatcherised faces, suggesting that rotation may affect normal and Thatcherised faces in similar ways. This is in contrast to the findings of Boutsen and Humphreys (2003) with same-face pairs which showed that although RTs were slower for (upright) Thatcherised pairs, they were not significantly reduced by inversion.

Boutsen and Humphreys (2003) explained this lack of an inversion effect with Thatcherised faces by arguing that inverting the eyes and mouth of a normal face disrupts configural information to the same extent as face inversion. The matching of upright Thatcherised faces is therefore as impaired as the matching of inverted (normal or Thatcherised) faces, so no inversion effect is observed. However, studies such as that of Schwaninger and Mast (2005) have shown almost equivalent RTs and accuracy scores for upright configurally distorted as for featurally distorted faces on 'same' trials (when the two faces were identical); and Carbon et al (2005) and Carbon and Leder (2005) have also found effects of inversion for Thatcherised faces in recognition tasks. Carbon et al (2005) found an inversion effect for Thatcherised faces for the N170 ERP amplitude for familiar-face recognition, whereas Carbon and Leder (2005) found a similar effect for RTs in a recognition task over long (but not short) presentation times. Therefore, it may be that in a simultaneous matching task with same-person pairs, a featural strategy is encouraged, and this is the case for both normal and Thatcherised face pairs. It is important to note, however, that other methodological differences may also account for this difference between the two studies. The difference may be due, for example, to the differential salience of the feature inversion (used to produce the Thatcherised faces) between the two studies. It is possible that the Thatcherised faces were harder to detect or less discriminable from normal faces in the Boutsen and Humphreys (2003) study, which may have given rise to the longer RTs for upright Thatcherised faces.

Nevertheless, as they stand, our findings are consistent with the predictions of the mental-rotation hypothesis. When there is no change to be detected (same trials), the hypothesis predicts that there will be no difference between normal and Thatcherised face pairs, because trials always contain identical stimuli, and participants would mentally rotate both types of stimuli to determine that they were the same. On different trials, however, rotation should have a substantial effect on both accuracy scores and RTs, and again this pattern of results was observed. The findings are also largely consistent with the view that a same-person matching task, when the two faces are identical, encourages a feature-based matching strategy, and that this is the case for both normal and Thatcherised faces. 


\section{Experiment 2}

Boutsen and Humphreys (2003) also looked at the matching of normal and Thatcherised faces in different-person pairs, where the two faces depicted different individuals, as a means of encouraging a more configural processing strategy to be employed. The task was again to decide as quickly and as accurately as possible whether the two faces were of the same type (both normal or both Thatcherised) or different (one normal and one Thatcherised). For same-type pairs an interaction was found between orientation and face type; normal faces again showed an inversion effect, but Thatcherised faces were responded to faster in the inverted condition than in the upright condition. There were no main effects of face type or of orientation. For different pairs, responses to inverted faces were significantly slower than those to upright faces. A similar pattern of results was found with different-person face parts (experiment 2). The fact that the additional face context did not influence or facilitate configural encoding when participants cannot rely on image-based matching suggests that local configural processing was used in this task, and that this information is disrupted by inversion (for normal faces) and by Thatcherisation (for upright faces only). Again, Boutsen and Humphreys (2003) suggested that this inversion effect for normal but not for Thatcherised faces highlights a qualitative difference in the encoding of these two types of faces.

Here we use the same paradigm as in experiment 1 to look at the effect of rotation on the matching of different-person pairs with normal and Thatcherised faces to compare the encoding strategies of these types of faces when a configural encoding strategy is encouraged. Boutsen and Humphreys (2003) argued that this task encouraged local configural processing of featural information for normal faces, but global configural processing - of features and their relationship to the whole-face context-for Thatcherised faces. For upright Thatcherised faces, the face context is incompatible with the Thatcherised parts, disturbing configural processing and producing longer RTs than for inverted Thatcherised faces. If there is a qualitative difference in the encoding of normal and Thatcherised faces on this task, we might expect similar results to those of Boutsen and Humphreys (2003); an interaction between face type and orientation, with a positive effect of inversion for Thatcherised faces, and an inversion effect for normal face matching. However, Carbon et al (2005) and Carbon and Leder (2005) have both shown effects of inversion for Thatcherised faces, with significant differences in RTs (and N170 amplitudes) between inverted and normal Thatcherised faces. Though there are a number of differences between our study and those of Carbon and colleagues, in light of the findings from experiment 1 , it is possible that we may again find different effects of Thatcherisation and rotation from those of Boutsen and Humphreys (2003). The mental-rotation hypothesis would predict equivalent effects of rotation for normal and Thatcherised faces for faces rotated up to $135^{\circ}$, but an advantage for inverted Thatcherised faces, because the local features do not have to be mentally rotated for recognition as they are already in an upright orientation.

\subsection{Method}

3.1.1 Design. The two within-participants independent variables were the orientation of the faces [upright $\left(0^{\circ}\right), 45^{\circ}, 90^{\circ}, 135^{\circ}$, and $180^{\circ}$ ] and whether the two faces were the same or different (both normal, both Thatcherised, or one normal and one Thatcherised). The dependent variables were the accuracy and latency of response to the same/different decision for same-face pairs.

3.1.2 Participants. Twenty undergraduates from Cardiff University with normal or correctedto-normal vision received course credits for their participation in this experiment. 
3.1.3 Materials. The stimuli detailed in experiment 1 were also used in this experiment. The six faces were used to create a set of face pairs, with each face paired with each other face once, making 15 pairs for each angle of rotation, 150 pairs for each type of response (same/different). Each face pair was repeated three times, making 900 trials in the experiment. On 'different' trials (when a normal and a Thatcherised face were paired together), the position of the normal face was counterbalanced between trials, such that it appeared on the left of the screen (and the Thatcherised face on the right) in half of the trials, and on the right of the screen (with the Thatcherised face on the left) in the other half to give the same number of 'same' and 'different' trials. All images were presented on a computer with Superlab.

3.1.4 Procedure. In each trial, participants saw two faces on the screen. These faces were either both normal, both Thatcherised, or one normal, and one Thatcherised. Participants were asked to indicate whether they thought the two images were of the same type (both normal or both Thatcherised) or different (one normal, one Thatcherised), by pressing one of two keys on a keyboard. In each trial, the face pair was presented until the participant made a response, followed by a $1 \mathrm{~s}$ blank interval before the next trial. Participants were asked to respond as quickly and accurately as possible.

\subsection{Results}

The results were calculated in the same way as in experiment 1 . The mean RTs and percentage of errors for same pairs (both normal or both Thatcherised) are shown in figures 6 and 7, respectively. The mean percentage of RTs which were excluded for being more than three standard deviations from the mean was $1.2 \%$ for normal face pairs and $2.5 \%$ for Thatcherised face pairs.
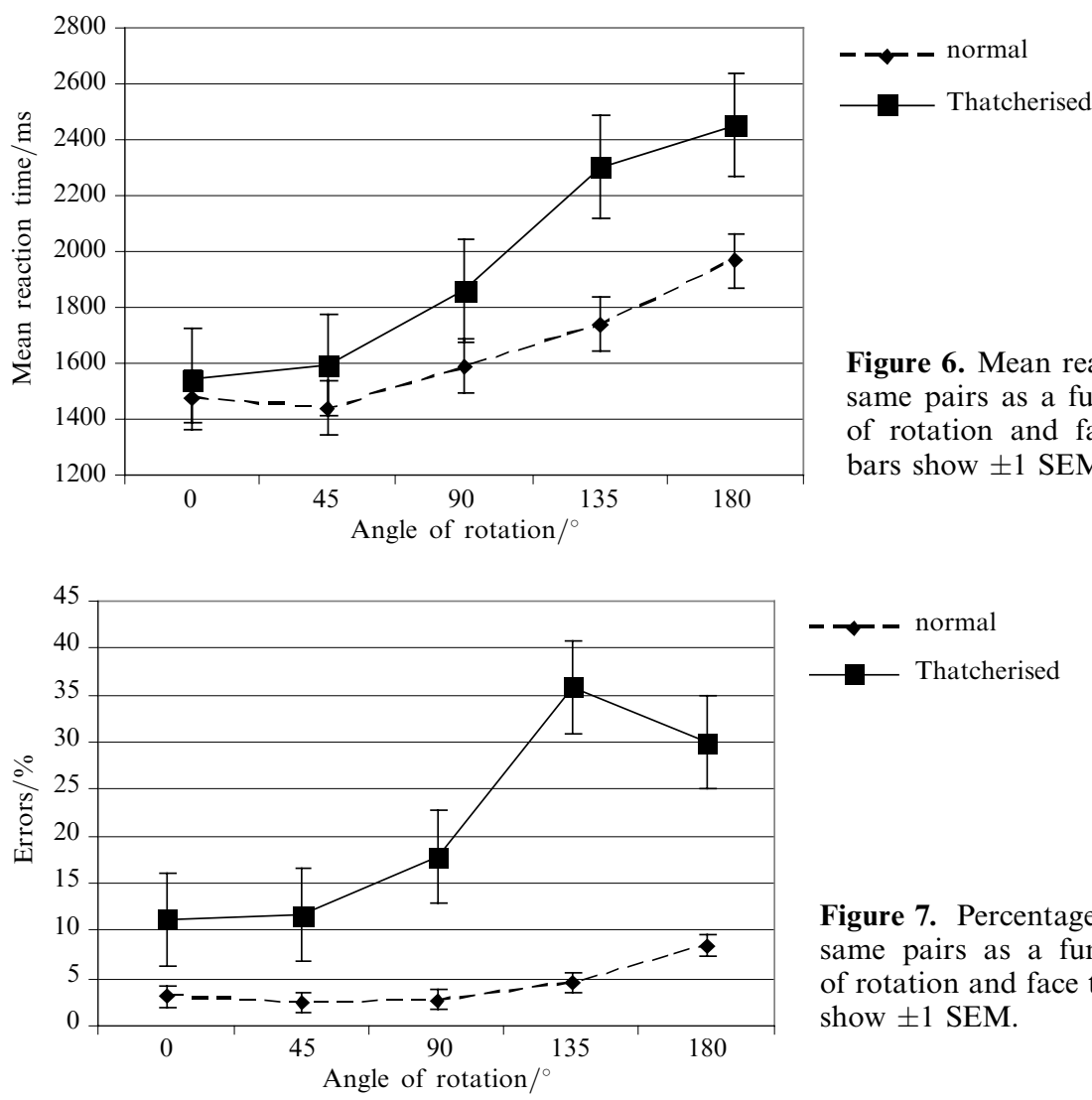

Figure 6. Mean reaction times for same pairs as a function of angle of rotation and face type. Error bars show \pm 1 SEM.

Figure 7. Percentage of errors for same pairs as a function of angle of rotation and face type. Error bars show \pm 1 SEM. 
A repeated-measures ANOVA was conducted on the mean RTs shown in figure 6. There was a significant effect of orientation on performance $\left(F_{4,76}=15.703, p<0.05\right)$, a significant effect of face type $\left(F_{1,19}=44.046, p<0.05\right)$, and a significant interaction between orientation and face type $\left(F_{4,76}=7.696, p<0.05\right)$. There was no significant difference between upright normal and upright Thatcherised faces $\left(t_{19}=0.982\right.$, $p>0.05)$, but significant differences emerged between normal and Thatcherised faces at all other angles of rotation ( $p<0.05$ in all cases). For normal faces there was no significant difference between upright and $45^{\circ}$ or upright and $90^{\circ}$ rotated faces $\left(t_{19}=0.733, p>0.05\right.$ and $t_{19}=1.801, p>0.05$, respectively), but significant differences emerged between upright and $135^{\circ}$ rotated and upright and inverted faces $\left(t_{19}=2.670, p<0.05\right.$ and $t_{19}=3.647, p<0.05$, respectively). There were significant differences between $45^{\circ}$ and $90^{\circ}, 90^{\circ}$ and $135^{\circ}$, and $135^{\circ}$ and $180^{\circ}$ rotated faces ( $p<0.05$ in all cases). For Thatcherised faces, there was no significant difference between $0^{\circ}$ and $45^{\circ}$ rotated faces $\left(t_{19}=1.190, p>0.05\right)$, but significant differences were found between upright and all other angles of rotation $(p<0.05$ in all cases). Significant differences were also found between $45^{\circ}$ and $90^{\circ}$, and $90^{\circ}$ and $135^{\circ}$ rotated faces $\left(t_{19}=5.247, p<0.05\right.$ and $t_{19}=2.911, p<0.05$, respectively), but not between $135^{\circ}$ and $180^{\circ}\left(t_{19}=1.370, p>0.05\right)$.

A repeated-measures ANOVA conducted on the error data in figure 7 also showed significant effects of rotation $\left(F_{4,76}=24.637, p<0.05\right)$, face type $\left(F_{1,19}=114.553\right.$, $p<0.05)$, and an interaction between face type and rotation $\left(F_{4,76}=18.939, p<0.05\right)$, again showing that the effect of rotation was much greater for Thatcherised than for normal face pairs. The difference between normal and Thatcherised faces was significant at all angles of rotation ( $p<0.05$ in all cases). For normal faces, only inverted faces differed significantly from upright faces $\left(t_{19}=3.336, p<0.05\right)$. Significant differences were also found between $90^{\circ}$ and $135^{\circ}$ rotated faces, and $135^{\circ}$ rotated and inverted faces $\left(t_{19}=2.203, p<0.05\right.$ and $t_{19}=2.559, p<0.05$, respectively). For Thatcherised faces, there was no significant difference between upright and $45^{\circ}$ rotated faces $\left(t_{19}=0.376, p>0.05\right)$, but significant differences were found between upright and all other angles of rotation ( $p<0.05$ in all cases). In addition, significant differences were found between $45^{\circ}$ and $90^{\circ}$, and $90^{\circ}$ and $135^{\circ}$ rotated faces, but the decline in errors for Thatcherised face pairs rotated between $135^{\circ}$ and $180^{\circ}$ was not significant $\left(t_{19}=1.640, p=0.118\right)$.

The data for same pairs, therefore, show consistent effects of rotation. This effect was much greater for Thatcherised than for normal face pairs, and Thatcherised face pairs were consistently affected by rotations beyond $45^{\circ}$ from the upright, whereas normal face pairs were affected by $90^{\circ}$ rotations.

The mean RTs and percentage of errors for different pairs (one normal, one Thatcherised) are shown in figures 8 and 9, respectively. The mean percentage of RTs excluded for being more than 3 standard deviations from the mean was $1.6 \%$.

A repeated-measures ANOVA was conducted on the mean RTs shown in figure 8. There was a significant effect of rotation on performance $\left(F_{4,76}=16.983, p<0.05\right)$. There was a small increase in RTs for rotations between $0^{\circ}$ and $45^{\circ}$, with a larger increase between $45^{\circ}$ and $90^{\circ}$, and a paired-samples $t$-test revealed that this increase was significantly larger than that between $0^{\circ}$ and $45^{\circ}\left(t_{19}=2.847, p<0.05\right)$. The increase between $90^{\circ}$ and $135^{\circ}$ was also significant $\left(t_{19}=3.420, p<0.05\right)$, but not that between $135^{\circ}$ and $180^{\circ}$ $\left(t_{19}=1.812\right)$. This appears to suggest that when one is required to detect that one face in a non-identical pair is Thatcherised, rotation of the faces to $90^{\circ}$ makes this task significantly more time consuming, and this may indicate that a different processing strategy is being employed for faces rotated to this point.

A repeated-measures ANOVA of the error data in figure 9 also revealed a significant effect of rotation $\left(F_{4,76}=37.323, p<0.05\right)$ but, as in experiment 1 , showed an 

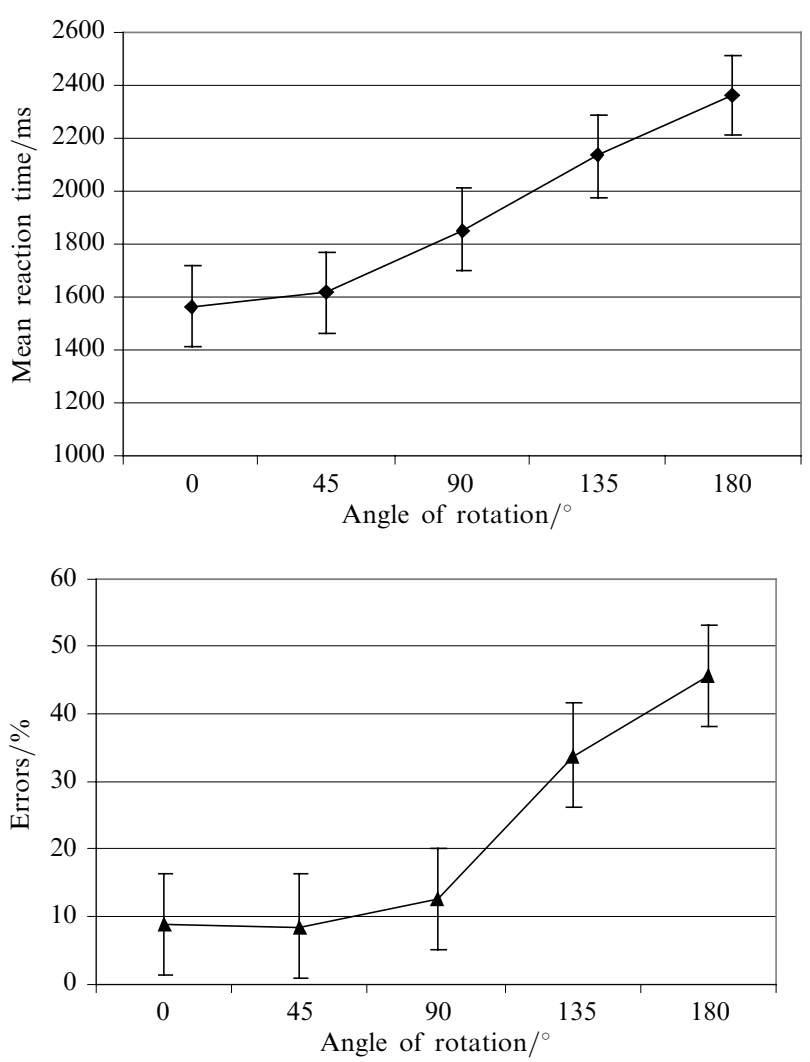

Figure 8. Mean reaction time for different pairs as a function of angle of rotation. Error bars show \pm 1 SEM.
Figure 9. Percentage of errors for different pairs as a function of angle of rotation. Error bars show \pm 1 SEM.

abrupt increase in errors for faces rotated beyond $90^{\circ}\left(t_{19}=5.888, p<0.05\right)$. The increase in errors between $135^{\circ}$ and $180^{\circ}$ was also significant $\left(t_{19}=4.663, p<0.05\right)$. These data appear to suggest that there is a clear point beyond which participants find it difficult to detect that the two faces are of a different type, providing further support for our findings from experiment 1.

\subsection{Comparison of experiments 1 and 2}

A between-experiments comparison was performed to see if there was any difference in the effect of rotation between same-person and different-person face pairs. Separate analyses were conducted for 'same' (collapsed across face type) and 'different' responses. A mixed ANOVA was conducted on the 'same' decision data, and showed a significant effect of rotation $\left(F_{4,312}=37.553, p<0.05\right)$, and a significant interaction between face identity and rotation $\left(F_{4,312}=6.679, p<0.05\right)$. The main effect of identity between the two experiments, however, was not significant $\left(F_{1,78}=0.715\right.$, $p>0.05)$. On investigating the interaction, a paired-samples $t$-test revealed that the effect of rotation was greater for different-identity pairs $(697 \mathrm{~ms})$ than for same-identity pairs (346 ms; $t_{39}=2.210, p<0.05$ ). For same-person pairs, a series of paired-sample $t$-tests showed significant differences between $0^{\circ}$ and $45^{\circ}, 45^{\circ}$ and $90^{\circ}, 90^{\circ}$ and $135^{\circ}$, but not $135^{\circ}$ and $180^{\circ}$ rotated faces $\left(t_{39}=0.264, p>0.05 ; p<0.05\right.$ in all other cases). For different-person pairs, only the difference between $0^{\circ}$ and $45^{\circ}$ rotated faces was not significant $\left(t_{59}=0.117, p>0.05\right)$; significant differences were found between all other angles of rotation.

A mixed ANOVA of the 'different' decision data for same-person and different-person pairs also showed a significant effect of rotation $\left(F_{4,152}=34.255, p<0.05\right)$. The interaction between rotation and experiment was not significant $\left(F_{4,152}=0.204, p>0.05\right)$. 
Here, the main effect of identity between the two experiments was significant $\left(F_{1,38}=4.196, p<0.05\right)$. A series of paired-sample $t$-tests revealed that the difference between same-person and different-person pairs emerged for upright $\left(t_{19}=2.259\right.$, $p<0.05)$ and $90^{\circ}$ rotated faces $\left(t_{19}=2.388, p<0.05\right.$ in all other cases). For sameperson pairs, significant differences were found between all angles of rotation between: $0^{\circ}$ and $45^{\circ} ; 45^{\circ}$ and $90^{\circ} ; 90^{\circ}$ and $135^{\circ}$; and $135^{\circ}$ and $180^{\circ}(p<0.05$ in all cases $)$. For different-person pairs, only the differences between $0^{\circ}$ and $45^{\circ}\left(t_{19}=1.291, p>0.05\right)$, and $135^{\circ}$ and $180^{\circ}\left(t_{19}=1.812, p>0.05\right)$ rotated faces were not significant.

In terms of the regression analyses, the data from the two experiments were combined for each condition (eg normal, Thatcherised, different), and linear and cubic functions were fitted to the data. Comparisons of the ability of the linear and cubic functions to account for the data showed that for normal face pairs a cubic function provided a better fit to the data than a linear function $\left(F_{2,1}=1422\right)$, but this was not the case in any of the other conditions. Thus, the overall effect of rotation in these experiments has been one of a roughly linear decline in performance as the face was rotated away from the upright, although there is some evidence of nonlinearities.

\subsection{Discussion}

The results of this experiment show a number of differences with our findings from experiment 1 and with the work of Boutsen and Humphreys (2003). Here, we found that the matching of Thatcherised faces yielded significantly longer RTs than did the matching of normal faces. This is in contrast to the findings from experiment 1 , and the Boutsen and Humphreys (2003) experiment with different-identity face pairs, which both showed no difference between normal and Thatcherised faces. We also found a significant effect of rotation, and an interaction between face type and rotation. Boutsen and Humphreys (2003) also reported such an interaction, but showed that inversion affected normal and Thatcherised faces in opposite directions; our results appear to suggest that the direction of the effect is the same, but rotation has a more detrimental effect on the matching of Thatcherised face pairs than normal face pairs. Thus, it would appear that Thatcherisation disproportionately disrupts the matching of face pairs when the task cannot rely on image-based matching. Furthermore, Thatcherised face pairs were consistently affected by rotation beyond $45^{\circ}$ from the upright, whereas normal face pairs were affected by $90^{\circ}$ rotations. The data from different trials were largely consistent with those of experiment 1, although different pairs were not significantly affected by $45^{\circ}$ rotations in this experiment. Nevertheless, when one is required to detect that one face in a non-identical pair is Thatcherised, rotation of the faces to $90^{\circ}$ makes this task significantly more time-consuming, and this may indicate that a different processing strategy is being employed for faces rotated beyond this point. There was an overall difference between same-person and different-person matching, with the latter producing longer RTs and a greater percentage of errors. The error data were also consistent with our previous findings, suggesting that there is a clear point (once the face is rotated beyond $90^{\circ}$ ) beyond which participants find it difficult to detect that the two faces are of a different type.

Comparing the two experiments, for 'same' responses there was an interaction between rotation and identity, with the effect of rotation being greater for differentidentity pairs than for same-identity pairs. Meanwhile, for 'different' responses the effect of rotation was of approximately the same magnitude for same and differentperson pairs, but different-person pairs produced consistently longer RTs and more errors, suggesting that this task is particularly difficult when matching cannot be purely image-based. We will now discuss the findings from these experiments, their differences (and similarities) with previous research, and the extent to which they can be explained by the mental-rotation hypothesis of face processing. 


\section{General discussion}

In these experiments we have attempted to look at the effects of rotation on configural information and to examine why configural information is difficult to retrieve from rotated faces. On a same-person matching task, which could be achieved by matching image-based featural information, an effect of rotation was observed for both normal and Thatcherised face pairs, and this effect was approximately the same for both types of faces. Thatcherised face pairs produced more errors than normal faces, however. On different-person trials, RTs became progressively longer with increasing rotation away from the upright, whereas accuracy scores were significantly affected by rotations beyond $90^{\circ}$. When the two faces depicted different people, an effect of rotation was again observed for normal and Thatcherised face pairs, but the effect was significantly greater for Thatcherised than for normal faces, and this was true both of RTs and of accuracy scores. On different trials, the data mirrored those of experiment 1 , with RTs increasing with rotation from the upright, and accuracy scores significantly affected by rotations beyond $90^{\circ}$. Both of these effects were more pronounced for different-person face pairs, and the effect of rotation was also greater for differentidentity pairs than for same-identity pairs for 'same' responses.

Perhaps the most surprising finding here, in light of previous research, is that of an inversion effect for Thatcherised face pairs, and this was apparent for both sameperson and different-person face matching. We argue that the mental-rotation hypothesis is able to account, at least in part, for this finding. When there is no change to be detected (same trials), the hypothesis predicts that there should be no difference between normal and Thatcherised face pairs, because trials always contain identical stimuli, and participants would mentally rotate both types of stimuli to determine that they were the same. The data from different trials were also consistent with the hypothesis, however with rotation conferring a substantial effect on both accuracy scores and RTs.

The pattern of findings for 'same' responses with different-person pairs, however, is more consistent with holistic processing being used to perform this task. Carbon and Leder (2005) noted that for inverted Thatcherised faces, although the eyes and mouth are seen in the correct orientation, the Gestalt of the overall image is not coherent. Normal inverted faces, in contrast, have a coherent Gestalt, but the eyes and mouth are inverted, which may hinder featural processing of these faces. They suggest that, if face processing is holistic in nature, one might expect inverted normal faces to be processed faster, because they have a more coherent holistic Gestalt than inverted Thatcherised faces. Consistent with this, they found that inverted Thatcherised faces were recognised faster than inverted normal faces at short presentation times, but this advantage for Thatcherised faces disappeared in the long presentation condition (200 ms), with inverted normal faces showing faster recognition times. Thus, when matching cannot be based on image-based featural information, participants may revert to a holistic processing strategy, which is able to account for the greater effect of rotation for Thatcherised than for normal face pairs. The data for 'different' responses, however, were again consistent with the mental-rotation hypothesis, which suggests that participants may rotate the two faces to confirm that they are indeed different.

We have also considered the question whether the relationship between rotation and face recognition is of a linear or nonlinear nature. Although we are unable to conclude that there is definitely a linear relationship between rotation and face-matching performance in these tasks, the majority of the data suggested that a linear function provided the best account of the data. These findings are in broad agreement with the third prediction of the mental-rotation hypothesis mentioned in the introduction: that recognition performance is dependent on the amount of normalisation required. Moreover, our findings provide an insight into the effect of rotation on face-recognition tasks, 
where previous studies reporting a nonlinear effect have looked at bizarreness ratings of Thatcherised faces, or the simple detection of a Thatcherised face.

Though only our study and that of Boutsen and Humphreys (2003) have looked at 'same' decisions in these types of matching types with Thatcherised faces (ie matching on the basis of image type), our findings are nevertheless consistent with other research, which shows that inversion is disruptive to the processing of Thatcherised faces (eg Murray et al 2000; Sturzel and Spillmann 2000; Lewis 2001). We propose an alternative explanation of the effect of configural information on face matching: that Thatcherisation disproportionately disrupts the matching of face pairs in a task which encourages configural processing (matching different-identity face pairs), but when matching can be image-based, as in experiment 1 , the loss of configural information through rotation is no greater for Thatcherised face pairs than for normal pairs. Our findings appear to suggest that there may not be a qualitative difference between the encoding of Thatcherised and normal faces as such, but that different tasks involve different processing or encoding strategies, and that, when configural encoding is required, Thatcherised faces are affected more by rotation than normal faces.

\section{References}

Bartlett J C, Searcy J, 1993 "Inversion and configuration of faces" Cognitive Psychology 25281 - 316

Boutsen L, Humphreys G W, 2003 "The effect of inversion on the encoding of normal and 'Thatcherized' faces" Quarterly Journal of Experimental Psychology A 56 955-975

Carbon C C, Leder H, 2005 "When feature information comes first! Early processing of inverted faces" Perception $341117-1134$

Carbon C C, Schweinberger S R, Kaufmann J M, Leder H, 2005 "The Thatcher illusion seen by the brain: An event-related brain potentials study" Cognitive Brain Research $24544-555$

Carey S, Diamond R, 1977 "From piecemeal to configurational representation of faces" Science $195312-314$

Corballis M C, Zbrodoff N J, Shetzer L I, Butler P B, 1978 "Decisions about identity and orientation of rotated letters and digits" Memory \& Cognition $698-107$

Diamond R, Carey S, 1986 "Why faces are and are not special: An effect of expertise" Journal of Experimental Psychology: General $115107-117$

Lewis M B, 2001 "The Lady's not for turning. Rotation of the Thatcher illusion” Perception 30 $769-774$

Lewis M B, Glenister T E, 2003 "A sideways look at configural encoding: Two different effects of face rotation" Perception 32 7-14

Lewis M B, Johnston R A, 1997 "The Thatcher illusion as a test of configural disruption" Perception $26225-227$

Murray J E, Yong E, Rhodes G, 2000 "Revisiting the perception of upside-down faces" Psychological Science $11498-502$

Rhodes G, Brake S, Atkinson A P, 1993 "What's lost in inverted faces?" Cognition 47 25-57

Rock I, 1973 Orientation and Form (San Diego, CA: Academic Press)

Schwaninger A, Mast F W, 2005 "The face-inversion effect can be explained by the capacity limitations of an orientation normalization mechanism" Japanese Psychological Research 47 $216-222$

Searcy J H, Bartlett J C, 1996 "Inversion and processing of component and spatial-relational information in faces" Journal of Experimental Psychology: Human Perception and Performance $22904-915$

Sergent J, 1984 "An investigation into component and configural processes underlying face perception" British Journal of Psychology 75221 - 242

Shepard R N, Metzler J, 1971 "Mental rotation of three-dimensional objects" Science 171701 - 703

Sturzel F, Spillmann L, 2000 "Thatcher illusion: Dependence on angle of rotation" Perception 29 $937-942$

Thompson P, 1980 "Margaret Thatcher: A new illusion" Perception 9 483-484

Valentine T, Bruce V, 1988 "Mental rotation of faces" Memory \& Cognition $16556-566$

Yin R K, 1969 "Looking at upside-down faces" Journal of Experimental Psychology 81 141-145 


\section{PERTEPTION}

VOLUME 362007

www.perceptionweb.com

Conditions of use. This article may be downloaded from the Perception website for personal research by members of subscribing organisations. Authors are entitled to distribute their own article (in printed form or by e-mail) to up to 50 people. This PDF may not be placed on any website (or other online distribution system) without permission of the publisher. 